\title{
Work-Life-Balance und Praxisgründung - passt das zusammen?
}

Grundsätzlich lässt sich diese Frage sehr leicht beantworten. Jede Minute, die man in der Anfangsphase in die Praxis investieren kann, macht Sinn und ist effektiver, als eine nachträgliche Justierung.

\section{Dr. Philipp F. Gebhardt // \\ BISS45 Kieferorthopädie Berlin, www.biss45.de \\ Dr. Gebhardt berichtet seit 2009 in jeder \\ Ausgabe über seinen Weg vom abge- \\ schlossenen Studium zum jungen Zahnarzt.

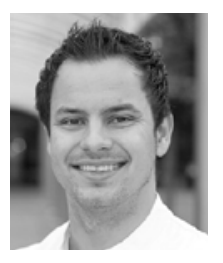

$\longrightarrow$

Schließen wir den Aspekt „Glück“ einmal aus, so ist Erfolg Simmer und unweigerlich mit Arbeit verbunden. Übernimmt man nun keine Praxis, in die man schon jahrelang als Angestellter bzw. Sohn/Tochter direkt oder indirekt Arbeit investiert hat, so sollte man einplanen, dass nach der regulären Arbeitszeit deutlich mehr Extrastunden notwendig werden. Ein stabiles familiäres bzw. privates Umfeld halte ich hier für essenziell, um Stressmomente nicht in das Privatleben zu transportieren. Sowohl aus finanziellen als auch aus logistischen Gründen lässt sich ein frisches Unternehmen nicht von Stunde null ideal aufstellen. Dabei kommt es regelmäßig und ungeplant zu Inte- rimsentscheidungen und etlichen Überstunden. Eine Ursache hierfür ist, dass viele Dinge, die man zum ersten Mal umsetzt, meist nicht zu $100 \%$ realisierbar sind oder nicht den eigenen Vorstellungen entsprechen. Dies reduziert sich zwar je nach Vorarbeit auf Kleinigkeiten und Fehler wie zum Beispiel eine falsche Telefonnummer auf den Visitenkarten, Druckfehler auf den Aufklärungsbögen, verbesserungsfähige Arbeitsverträge, eine unvollständige Homepage, falsche Druckereinstellungen, fehlerhafte Tischlerarbeiten ... Jedoch sind es gerade diese Kleinigkeiten, die sogar mehr Zeit kosten, als die großen Probleme.

Da die meisten Zahnmediziner sicherlich allein schon durch das Studium bedingt perfektionistisch veranlagt sind, führt dies zwangsläufig zu einer Extension der Überstunden. Leider verhält sich die Produktivität jedoch negativ proportional zu den täglichen Überstunden. Ich finde es hierbei essenziell, wenigstens ein bis zwei Stunden pro Woche einen Ausgleich wie zum Beispiel Sport zu finden. Nicht zuletzt aufgrund der in den letzten Ausgaben schon ausführlich erwähnten und sehr problematischen Lage auf dem Arbeitsmarkt bei den ZFA sollte man jedoch davon ausgehen, dass eine gesunde Zeitplanung schwer realisierbar ist. Je nach Ausmaß der Praxisgründung ist es sehr hilfreich, mit einer Anfangszeit von mindestens zwei Jahren zu rechnen, bevor es wieder möglich wird, zu gewohnten Arbeitszeiten zurückzufinden.

\section{Impressum}

Verlag: Springer-Verlag GmbH, Tiergartenstr. 17, 69121 Heidelberg,

Tel. +49 6221/487-0, www.springer.com

Geschäftsführer: Derk Haank, Martin Mos, Petrus W. J. Hendriks

Director Apotheke und Zahnmedizin: Marion Bornemann

Redaktionsleiterin Zahnmedizin: Susanne Redeker

Redaktionsleitung: Dr. Swanett Koops (Tel.-8217, swanett.koops@springer.com)

Layout: Arnulf Illing (Tel. -8952, arnulf.illing@springer.com)

Anzeigenleitung: Marita Säuberlich (Tel. -8309, Fax -68309,

marita.saeuberlich@springer.com),www.mediadaten.springermedizin.de

Corporate Publishing Zahnmedizin: Anja Weisse (Tel. -8739, Fax-68739, anja.weisse@

springer.com) (Adressen jeweils wie Verlag)

Druck: PHOENIX PRINT GmbH, Alfred-Nobel-Straße 33, 97080 Würzburg

Erscheinungsweise: 4 Ausgaben pro Jahr

Papierausgabe: ISSN 1869-5744, gedruckt auf säurefreiem Papier

Eigentümer \& Copyright: @ Springer-Verlag GmbH, Berlin, Heidelberg 2016. Die Sprin-

ger-Verlag GmbH ist Teil der Fachverlagsgruppe Springer Nature.

Die Zeitschrift sowie alle in ihr enthaltenen einzelnen Beiträge und Abbildungen sind

urheberrechtlich geschützt. Jede Verwertung, die nicht ausdrücklich vom Urheberrechtsgesetz zugelassen ist, bedarf der vorherigen schriftlichen Zustimmung des Verlags. Das gilt insbesondere für Vervielfältigungen, Bearbeitungen, Übersetzungen, Mikroverfilmungen und die Einspeicherung und Verarbeitung in elektronischen Systemen.

Preis für persönliche Abonnenten inkl. Online-Basis-Lizenz 2016:

(4 Hefte) EUR 58,98 (unverb. Preisempfehlung inkl. gesetzlicher MwSt.)

Einzelheftpreis 2016: EUR 23,- (unverb. Preisempfehlung inkl. gesetzlicher MwSt.).
Für Studierende und für Ärzte in der Facharztausbildung ermäßigt sich 2016 der Bezugspreis auf jährlich EUR 35,39 (unverb. Preisempfehlung inkl. gesetzlicher MwSt., Nachweis über Studium/Ausbildung erforderlich). Institutspreis inkl. Online-Basis-Lizenz 2016: (4 Hefte) EUR 199,09 (unverb. Preisempfehlung inkl. gesetzlicher MwSt.). Der Bezugspreis ist im Voraus zu zahlen. Das Abonnement kann bis 30 Tage vor Ende des Bezugszeitraums gekündigt werden.

Allgemeiner Hinweis zur gesetzlichen Mehrwertsteuer: Gedruckte Zeitschriften unterliegen grundsätzlich dem ermäßigten Steuersatz von 7\%, digitale Produkte (wie z.B. die Online-Version einer Zeitschrift) hingegen dem allgemeinen Steuersatz von 19\%. Die detaillierteAufteilung der einzelnen Mehrwertsteuer-Beträge entnehmen Sie bitte Ihrer Rechnung.

Bestellungen oder Rückfragen nimmt jede Buchhandlung oder der Verlag entgegen. Springer Customer Service Center GmbH, Haberstr. 7, 69126 Heidelberg,

Tel. +49 6221/345-4303, Fax +496221/345-4229, Leserservice@springer.com

(Mo.-Fr. 8.00 Uhr bis 18.00 Uhr)

Autoren können unter bestimmten Voraussetzungen an der Ausschüttung der Bibliotheks- und Fotokopietantiemen teilnehmen. Einzelheiten bei VG WORT, Abt. Wissenschaft, Goethestr. 49, 80336 München.

Angaben über Dosierungsanweisungen und Applikationsformen sind anhand anderer Literaturstellen oder der Packungsbeilage auf ihre Richtigkeit zu überprüfen. Der Verlag übernimmt keine Gewähr.

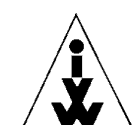

